was collected retrospectively from patient case notes, endoscopy reporting system and emergency theatre records.

Results 77 cases of AUGIB were identified with gender distribution of $56 \%$ males and $44 \%$ females. The median age of presentation was 67 years (range 20-93 years). Most cases of AUGIB (71\%) were acute admissions with the rest occurring among in-patients.

A major improvement in the service is that all patients had at least one endoscopy during their presentation with most endoscopies $(67 \%)$ performed within 24 hours or less and a further $(26 \%)$ carried out 2-7 days. Endoscopies were performed by gastroenterologist (66\%), surgeons (20\%) and specialist registrar (14\%). About a third $(27 \%)$ had out of hours $(\mathrm{OOH})$ emergency endoscopies and the remaining procedures were carried out in the dedicated inpatient lists. Majority (62\%) of the $\mathrm{OOH}$ procedures were done at the weekends.

A notable shortcoming was poor risk assessment (18\%) at presentation although retrospective risk scoring revealed a median Rockall Score of 3 (range 1-5). At presentation only $8 \%$ of patients were admitted to the dedicated gastroenterology ward before the first endoscopy while the majority (52\%) were managed initially on the acute medical wards and discharged or subsequently admitted to the gastroenterology ward.

Conclusion The introduction of a dedicated service has improved the management of AUGIB in our hospital serving a population of 325,000 particularly during the $\mathrm{OOH}$. The findings of this retrospective audit showed an AUGIB service collaborating medical gastroenterologists and surgeons is workable and sustainable in the setting of a district general hospital.

To further improve the service a dedicated AUGIB clerking proforma incorporating Rockall risk score assessment is being considered.

Disclosure of Interest None Declared.

\section{PTH-169 CLINICAL UTILITY OF A RAPID PCR ASSAY AND TRADITIONAL CCNA TO DETECT CLOSTRIDIUM DIFFICILE INFECTION (CDI) - COMPARISON TO CLINICAL DIAGNOSIS}

doi:10.1136/gutjnl-2013-304907.656

${ }^{1} \mathrm{~N}$ Berry, ${ }^{2} \mathrm{~B}$ Sewell, ${ }^{3, *} \mathrm{~S}$ Jafri, ${ }^{\prime} \mathrm{S}$ Vagia, ${ }^{3} \mathrm{C}$ Puli, 'E Rees, ${ }^{1} \mathrm{~A}$ Lewis, ${ }^{1} \mathrm{M}$ Isaac, ${ }^{3} \mathrm{C} \mathrm{L}$ Ch'ng. 'Microbiology ABM, Singleton Hospital, Public Health UK Microbiology ABM; ${ }^{2}$ College of Human and Health Sciences, Swansea University; ${ }^{3}$ Gastroenterology, ABM University Health Board, Swansea, UK

Introduction Clostridium difficile causes nosocomial antibiotic associated diarrhoea, with a range of mild to severe disease, pseudomembranous colitis, toxic megacolon and potentially fatal outcome. The diagnosis of this disease in clinical laboratories has traditionally been performed by cell culture cytotoxin neutralisation assay (CCNA) or by toxin $\mathrm{A} / \mathrm{B}$ detection using EIA. The routine test in our laboratory is CCNA, which takes 24-48 (-72) hours; it is labour intensive, requires specialist facilities, expertise and is not done out of hours. There are other rapid tests now available including GDH as well as molecular (real-time PCR) assays.

Methods This study was designed to assess the clinical relevance of a fully automated, random access PCR assay, Xpert C. difficile, for rapid identification of $C$. difficile infection (CDI) in comparison to clinical diagnosis as the reference method. During March to September 2011, 1040 samples from inpatients in 2 hospitals, with suspected CDI, were prospectively tested by routine cell culture cytotoxin neutralisation assay (CCNA), PCR (GeneXpert, Cepheid), and a GDH/Toxin EIA (Premier, Launch Diagnostics). Cytotoxicity was assessed after 24 and 48 hours. All PCR positive patients (and controls) were reviewed by a multidisciplinary team (Gastroenterologist, Microbiologist, infection control nurse, requesting staff).

Results C. difficile detection rates were $10.8 \%$ (PCR), $6 \%$ (CCNA) and $13.8 \%$ (GDH). 974/1035 (94.1\%) samples showed concordant
CCNA and PCR results, 89\% (886/985) were concordant for CCNA, PCR and GDH and 94.4\% (930/985) showed concordance between GDH and PCR. With clinical diagnosis as a reference, PCR was $99.1 \%$ sensitive, 98.9\% specific, with PPV 91.9\% and NPV 99.9\%. Surprisingly, CCNA on a single sample was only $51 \%$ sensitive, $99.4 \%$ specific, PPV was $91.9 \%$, NPV $94.3 \%$. GDH sensitivity was $83.8 \%$, specificity $94.5 \%$, PPV $64.7 \%$ and NPV $97.9 \% .59$ more samples were positive by PCR than CCNA (62); 54/59 were clinically CDI.

Conclusion We found PCR to be a more sensitive method than CCNA and GDH (sensitivity 83.8\%) for the detection of C.difficile infection (CDI). In contrast to using CCNA or an algorithm that includes GDH, the use of Xpert C.difficile PCR allows us to provide accurate and rapid (mostly same day) results to the clinicians.

Disclosure of Interest N. Berry Grant/Research Support from: Non promotional Educational research grant from Cepheid, B Sewell Grant/Research Support from: Non promotional Educational research grant from Cepheid, S. Jafri Grant/Research Support from: Non promotional Educational research grant from Cepheid, S Vagia Grant/Research Support from: Non promotional Educational research grant from Cepheid, C. Puli Grant/Research Support from: Non promotional Educational research grant from Cepheid, E. Rees Grant/Research Support from: Non promotional Educational research grant from Cepheid, A. Lewis Grant/Research Support from: Non promotional Educational research grant from Cepheid, M. Isaac Grant/Research Support from: Non promotional Educational research grant from Cepheid, C. L. Ch'ng Grant/Research Support from: Non promotional Educational research grant from Cepheid

\section{PTH-170 FIVE YEAR FOLLOW UP OF PATIENTS WITH ASYMPTOMATIC IRON DEFICIENCY ANAEMIA FOLLOWING NORMAL INVESTIGATIONS IN A NURSE LED PROTOCOL DRIVEN PATHWAY}

doi:10.1136/gutjnl-2013-304907.657

1., S A Townsend, '2D Cheung, 'R Ransford, 'E Horne, '1 Burdsall, 'H Griffiths. 'Gastroenterology, Hereford County Hospital, Hereford; 'astroenterology, Sandwell Hospital, Birmingham, UK

Introduction Systematic investigation of patients with iron deficiency anaemia has been proven to yield a $12 \%$ diagnosis for colorectal cancer and $5 \%$ for coeliac disease ${ }^{1}$. Following publication of BSG guidelines, a nurse led protocol driven pathway was established at a district general hospital.

We report the 5-year outcome of 122 patients with normal investigations entered onto this pathway and assess its efficacy in identifying GI pathology. This is the largest study to date in this field, and no other nurse led 5 year follow up studies have been published.

Methods Between 2001 and 2004, 271 cases of asymptomatic iron deficiency anaemia were referred to the pathway and 212 met the inclusion criteria. From initial investigations, $43 \%$ were diagnosed with an underlying cause of anaemia and we have now followed up the remaining 122 patients for a minimum of 5 years.

Data collected at presentation included: haemoglobin, mean cell volume, ferritin, creatinine, iron-binding capacity, CRP, drug use (aspirin, clopidogrel, warfarin, NSAIDs), co-morbidities, smoking status, BMI, and the results of investigations carried out (gastroscopy, colonoscopy, barium enema). Iron supplementation was recorded and haemoglobin level at 3 months.

Patients were divided into 2 groups: those that died or developed malignancy and those that were well at 5 years. The data was analysed for significant differences between the 2 groups and to identify risk factors for poor prognosis.

Results Analysis of primary and secondary care records generated outcome data of $97 \%$ (118) patients. $69 \%$ were female and $31 \%$ were male, mean age of 69 years. 